\title{
Réflexions sur les effets des nuisances chimiques et radiologiques en milieu professionnel*
}

\author{
J. LAFUMA ${ }^{\star \star}$ \\ (Manuscrit reçu le 7 février 1986)
}

En 1981, le gouvernement français a décidé de créer un comité scientifique chargé de proposer des valeurs limites pour les produits chimiques. Afin de profiter de l'expérience acquise dans le domaine nucléaire, un spécialiste des nuisances radiologiques a été associé aux travaux. La confrontation des points de vue a mis en évidence des différences fondamentales entre les deux types de nuisances.

Tout d'abord il existe, dans le domaine radiologique, un consensus international sur les valeurs des limites d'exposition, ce qui minimise considérablement les contraintes économiques. De plus, il existe une distinction claire entre les travailleurs et les membres du public.

Dans le domaine chimique, il n'existe pas de consensus international. Chaque pays peut avoir ses propres limites. La seule exception est celle des Communautés qui cherchent à unifier les valeurs limites parmi les Etats membres. Les contraintes économiques sont très lourdes. Un seul organisme scientifique international a une certaine autorité dans le domaine des cancérigènes: c'est le Centre international de recherche contre le cancer. Son rôle, toutefois, se limite à identifier les substances susceptibles d'avoir des effets cancérigènes.

Les législations les plus développées sont celles des USA et de l'URSS. Aux USA, les limites sont proposées par l'ACGIH (American conference of governmental industrial hygienists), et le NIOSH (National institute for occupational safety and health) et fixées par l'OSHA (Occupational safety and health administration).

Les valeurs proposées par l'ACGIH sont basées sur des études épidémiologiques mettant en valeur des dommages subis. L'OSHA fait intervenir, en plus, dans certains cas l'expérimentation animale. En URSS, les valeurs limites sont basées sur la détection d'effets. Elles sont donc, en général, plus faibles que les valeurs américaines.

* Communication présentée au Congrès franco-italien "Les expositions professionnelles - aspects méthodologiques et expériences pratiques", organisé à Nice du 29 au 31 janvier 1986 par la Société française de radioprotection et l'Associazione italiana di protezione contro le radiazioni.

** Commissariat à l'énergie atomique, Institut de protection et de sûreté nucléaire. Département de protection sanitaire, BP 6. 92265 Fontenay-aux-Roses Cedex. 
Dans le domaine chimique, la distinction entre travailleurs et membres du public est plus floue. En effet, si les produits de base purs sont élaborés dans de grosses sociétés, ils sont ensuite mélangés pour former des produits commerciaux dont l'emploi peut être dispersé entre de très petites sociétés (artisans) ou même parmi les membres du public (bricolage). L'effort de protection est également très différent selon que l'on s'adresse à de grosses sociétés, dans lesquelles la qualité de la protection est comparable à celle qui existe dans le nucléaire, ou que le produit est utilisé dans de petites entreprises.

Les valeurs limites, en outre, n'ont été établies que pour les produits purs et non pour les mélanges commerciaux.

Les effets dont on cherche à se protéger sont comparables dans les industries radiologiques et chimiques. Dans les deux cas, il existe des effets dont l'intensité dépend de la dose et pour lesquels il existe des seuils (qualifiés de non stochastiques en protection radiologique) et des effets dont la probabilité d'apparition - et non la sévérité - dépend de la dose (effets stochastiques). Cependant, les effets "non stochastiques" sont très différents dans les deux cas. La signification des limites de dose n'est donc pas la même.

Il existe dans l'industrie chimique des risques à très court terme, pouvant aller jusqu'à la mort, pour lesquels il a fallu fixer des valeurs "plafonds" qui ne doivent jamais être dépassées. C'est le cas pour l'oxyde de carbone (CO) ou l'oxyde cyanhydrique $(\mathrm{CHN})$. Ce sont des valeurs plafonds appelées VLE (valeurs limites d'exposition). Le coefficient de sécurité entre la valeur limite et la concentration toxique est, en général, très faible. De telles limites n'existent pas en protection radiologique.

Pour les effets non stochastiques à moyen terme, on utilise en chimie des valeurs moyennes d'exposition (VME). La moyenne doit être respectée sur une durée de $8 \mathrm{~h}$ correspondant à la journée de travail. Pour éviter d'atteindre la zone des effets à court terme, à ces VME est, en général, associée une VLE ou valeur limite d'exposition pendant une courte période fixée, en général, à $15 \mathrm{~min}$. Ce type de limitation n'existe pas en protection radiologique.

II existe, dans l'industrie chimique comme dans l'industrie nucléaire, des effets non stochastiques à long terme (bérylliose, silicose par exemple). Le concept des valeurs moyennes établies sur des périodes de l'ordre de l'année, tel qu'il est utilisé dans la protection radiologique, n'est pas utilisé dans le domaine chimique, probablement par crainte que la "dose" annuelle distribuée sur des temps courts n'entraîne l'apparition de lésions dues à des effets à court terme. Cette crainte est parfaitement fondée lorsque l'on voit que la dose limite radiologique trimestrielle de l'uranium naturel, absorbée en un seul jour, entraînerait, à coup sur, l'apparition d'une néphrite aiguë.

En toxicologie chimique, comme dans le domaine radiologique, l'action cancérigène est toujours accompagnée d'un autre type de toxicité. Mais, en protection chimique ce n'est pas toujours l'effet cancérigène qui impose la limite la plus basse. La limite pour le béryllium reste basée sur la protection de la bérylliose. Le béryllium est un cancérigène "faible" et le respect de la limite bérylliose entraîne une probabilité d'apparition de cancers pulmonaires inférieure à celle que l'on accepte en protection radiologique. 
Les différences qui existent entre les toxiques chimiques et les rayonnements sont encore amplifiées par les conditions de leurs emplois. Dans le domaine radiologique, on est en face d'un seul toxique : les rayonnements ionisants. Même des différences dans la physico-chimie des radioéléments, qui modifient les métabolismes, ne changent pas la nature du risque. On peut donc additionner des doses d'origines différentes. Le problème des synergies n'existe qu'entre les rayonnements et les produits chimiques et, dans les conditions réelles du travail, ne se pose que pour les effets stochastiques.

En chimie, les problèmes sont totalement différents. Les effets changent selon les différentes formes physico-chimiques. Certains effets ne sont observés que dans un type de poste de travail. La limite n'est donc valable que pour cette seule condition d'emploi. Le nickel, par exemple, n'a montré d'action cancérigène chez l'homme que pendant une étape du traitement du minerai : le grillage des mattes. On ne peut, d'ailleurs, affirmer qu'il s'agisse du nickel et non de certains de ses composés. En outre, le problème des additivités des doses est insoluble, car les effets sont différents. II en va de même pour celui des synergies, surtout quand on constate qu'en règle générale, les travailleurs dans l'industrie chimique ne sont jamais soumis à une seule nuisance au cours de leur vie professionnelle.

Si l'on compare la qualité des estimations de risques dans la protection radiologique et en toxicologie chimique, on observe, là encore, des différences importantes.

En protection radiologique, on a pu consacrer les efforts à une seule nuisance : les rayonnements ionisants. En épidémiologie, on a donc rassemblé des données importantes provenant d'expositions professionnelles anciennes, de nombreuses cohortes de malades irradiés à des fins diagnostiques ou thérapeutiques et, enfin, des survivants d'Hiroshima et Nagasaki. Si pour les expositions professionnelles anciennes, la dosimétrie est très approximative, elle est plus précise pour les malades et les survivants des bombardements atomiques. II est aujourd'hui raisonnable de penser qu'au niveau de la limite annuelle de dose $(0,05 \mathrm{~Sv})$, l'estimation de risque est suffisamment correcte pour les besoins de la protection. L'expérimentation animale, largement utilisée, a permis de préciser les coefficients correctifs à utiliser pour tous les types d'exposition pour lesquels les données humaines sont trop fragmentaires ou inexistantes (neutrons, plutonium,...).

En toxicologie chimique, la multiplicité des toxiques n'a pas permis la concentration des efforts. L'épidémiologie, notamment, est très dispersée. Pour les effets aigus, on dispose de données qui ne sont que des "cas cliniques", en très petit nombre pour un même toxique. Pour les effets chroniques, à l'exception de la silicose et des effets associés à l'amiante pour laquelle on dispose de très nombreuses données, les enquêtes épidémiologiques sont limitées et en très petit nombre pour un même toxique. L'expérimentation animale, de très bonne qualité, est très dispersée. En cancérologie, l'effort a davantage porté sur la détection du caractère "cancérigène" que sur l'établissement de relations dose-effets précises. Si la connaissance de la capacité cancérigène est précieuse pour les indemnisations, elle n'apporte rien pour la fixation de limites d'exposition. 
Malgré ces difficultés, on peut dire que la qualité des estimations de risque et des valeurs limites qui en découlent est bonne pour les effets à court et moyen terme. Pour les effets à long terme, comme l'induction des cancers, les données trop fragmentaires n'ont pas toujours permis de fixer des valeurs limites précises. Mais, lorsque la qualité des données est suffisante, la sécurité des limites de doses est à un niveau comparable à celui qui existe en protection radiologique.

\section{CONCLUSION}

On peut dire que les problèmes posés à la protection radiologique sont beaucoup plus simples que ceux que rencontre la protection dans l'industrie chimique. Les difficultés se retrouvent aussi bien pour la fixation des limites d'exposition que pour la surveillance des travailleurs qui inhalent très souvent des atmosphères contenant plusieurs contaminants dont la nature chimique peut être très différente. II reste, en protection chimique, un très grand nombre de problèmes à résoudre. L'acquis de la protection radiologique pourra, sur certains points, apporter une aide précieuse, mais on ne pourra pas, dans l'industrie chimique, prendre la protection radiologique comme modèle unique. 